Recherche Médicale "Equipe FRM DEQ2014039529", the French Ministry of Research (Agence Nationale pour la Recherche ANR-17-CE16-0008 and the Investissements d'Avenir programme ANR-11-IDEX-0004-02) and the DIM Cerveau et Pensée région lle-de-France (PME2012). L.M.'s team is part of the École des Neurosciences de Paris lle-de-France network and of the Bio-Psy Labex and as such this work was supported by French state funds managed by the ANR within the Investissements d'Avenir programme under reference ANR-11-IDEX-0004-02.Competing interests: The authors declare no competing interests.

Publisher's note: Springer Nature remains neutral with regard to jurisdictional claims in published maps and institutional affiliations.

\section{REFERENCES}

1. Richardson-Jones JW, Craige CP, Guiard BP, Stephen A, Metzger KL, Kung HF, et al. 5-HT1A autoreceptor levels determine vulnerability to stress and response to antidepressants. Neuron. 2010;65:40-52.
2. Doly S, Valjent E, Setola V, Callebert J, Herve D, Launay JM, et al. Serotonin 5-HT2B receptors are required for 3,4-methylenedioxymethamphetamine-induced hyperlocomotion and 5-HT release in vivo and in vitro. J Neurosci. 2008;28:2933-40.

3. Diaz SL, Doly S, Narboux-Nême N, Fernandez S, Mazot P, Banas S, et al. 5-HT2B receptors are required for serotonin-selective antidepressant actions. Mol Psychiatry. 2012;17:154-63.

4. Belmer A, Quentin E, Diaz SL, Guiard BP, Fernandez SP, Doly S, et al. Positive regulation of raphe serotonin neurons by serotonin $2 \mathrm{~B}$ receptors. Neuropsychopharmacology. 2018;43:1623-32.

5. Bevilacqua L, Doly S, Kaprio J, Yuan Q, Tikkanen R, Paunio T, et al. A populationspecific HTR2B stop codon predisposes to severe impulsivity. Nature. 2010;468:1061-6.

6. Colgan LA, Cavolo SL, Commons KG, Levitan ES. Action potential-independent and pharmacologically unique vesicular serotonin release from dendrites. J Neurosci. 2012;32:15737-46.

\title{
Dynamic network targeting for closed-loop deep brain stimulation
}

\author{
Alexander B. Herman ${ }^{1}$ and Alik S. Widge (iD ${ }^{1}$ \\ Neuropsychopharmacology (2019) 44:219-220; https://doi.org/10.1038/s41386-018-0210-x
}

Deep brain stimulation (DBS) has gained wide use in movement disorders and remains an area of active research in psychiatric disorders. Recent clinical trial setbacks may reflect clinicians' trial-and-error approach to selecting stimulation parameters [1]. Newer studies focus on "closed-loop" approaches, where DBS settings are adjusted based on an objective, brain-based readout. Those studies divide roughly into "anatomical" approaches based on targeting stimulation to specific white matter (WM) bundles and "physiologic" approaches that focus on changing pathologic signatures in brain electrical activity. We argue that success in psychiatric applications may require a synthesis of both approaches: individualized anatomically guided electrode placement coupled with biomarker-responsive targeting of network dynamics.

The anatomical approach optimizes clinical response by tailoring electrode placement relative to individual brain anatomy. In subcallosal cingulate (SCC) DBS for depression, an open-label study prospectively targeted the intersection of four different tracts in peri-SCC WM using individualized probabilistic tractography. Out of 11,9 patients responded, a substantial improvement over the same group's prior results [2]. The same approach is now being expanded beyond SCC WM to optimize DBS placement in the ventral striatum/ventral capsule and medial forebrain bundle [1].

In comparison, the physiology-based closed-loop DBS approach is making strides in neurological disorders. Clinical outcomes improve in Parkinson disease when DBS is targeted to suppress specific cortical electrical oscillations or is locked to the phase of those oscillations [3]. A similar oscillatory feature was successfully used as a control signal in responsive DBS for Tourette syndrome [4]. Closed-loop DBS-like stimulation has also enhanced human memory. Recordings from sites across the brain can predict periods of poor memory encoding, and lateral temporal stimulation at those timepoints rescues memory performance [5]. If similar biomarkers can be identified for psychiatric symptoms, an analogous responsive stimulation approach should be possible in mental disorders. Preliminary evidence suggests that such biomarkers can be identified through a focus on cross-diagnostic domains of function, and that those markers can in turn be used for closedloop control of psychiatrically relevant functions such as emotion regulation [6].

Psychiatric disorders likely involve dysfunction across multiscale neural networks [1,7], and effective DBS appears to require modulation of multiple circuits [2]. These results suggest the potential power of a multinode, network approach to sensing and stimulating in DBS. Delivering stimulation in response to features on multiple time scales (for instance, both amplitude and phase) may increase symptom relief while reducing side effects [3]. In conditions with distributed pathology, recording from and stimulating multiple areas simultaneously may better control network interactions. The ability to sense multiple network nodes might allow a better assessment of stimulation's effects on network connectivity/ activity. Conversely, network activity might best be modulated by multisite stimulation. DBS and related technologies are believed to act by de-synchronizing brain networks, but this depends on stimulation efficiently propagating within those networks. In cases where single-site stimulation fails to

${ }^{1}$ Department of Psychiatry, University of Minnesota, Minneapolis, MN, USA

Correspondence: Alik S. Widge (awidge@umn.edu)

Received: 28 July 2018 Revised: 26 August 2018 Accepted: 28 August 2018

Published online: 18 September 2018 
adequately drive "downstream" nodes, a second stimulating site could enhance the network disruption. Fusing the anatomic and physiologic approaches into a dynamic, network-targeted approach to closed-loop DBS may be the next horizon for personalized treatment in severe psychiatric disorders.

\section{ACKNOWLEDGEMENTS}

A.S.W. acknowledges support from the OneMind Institute, National Institutes of Health (contracts MH10972, MH111320, NS100548, and MH113103), MnDRIVE Brain Conditions initiative, and MnDRIVE Medical Discovery Team on Addictions. A.B.H. acknowledges support from the MnDRIVE Brain Conditions initiative.Competing interests: The authors declare no competing interests.

Publisher's note: Springer Nature remains neutral with regard to jurisdictional claims in published maps and institutional affiliations.

\section{REFERENCES}

1. Widge AS, Malone DA Jr., Dougherty DD. Closing the loop on deep brain stimulation for treatment-resistant depression. Front Neurosci. 2018;12:640-10.

2. Riva-Posse P, Choi KS, Holtzheimer PE, Crowell AL, Garlow SJ, Rajendra JK, et al. A connectomic approach for subcallosal cingulate deep brain stimulation surgery: prospective targeting in treatment-resistant depression. Mol Psychiatry. 2017;23:1-7.

3. Meidahl AC, Tinkhauser G, Herz DM, Cagnan H, Debarros J, Brown P. Adaptive deep brain stimulation for movement disorders: the long road to clinical therapy. Mov Disord. 2017;32:810-9.

4. Molina R, Okun MS, Shute JB, Opri E, Rossi PJ, Martinez-Ramirez D, et al. Report of a patient undergoing chronic responsive deep brain stimulation for Tourette syndrome: proof of concept. J Neurosurg. 2017 1-7 https://doi.org/10.3171/2017.6. JNS17626.

5. Ezzyat Y, Wanda PA, Levy DF, Kadel A, Aka A, Pedisich I, et al. Closed-loop stimulation of temporal cortex rescues functional networks and improves memory. Nat Commun. 2018;9:1-8.

6. Widge AS, Ellard KK, Paulk AC, Basu I, Yousefi A, Zorowitz S, et al. Treating refractory mental illness with closed-loop brain stimulation: progress towards a patient-specific transdiagnostic approach. Exp Neurol. 2017;287:361-72.

7. Vinogradov S, Herman A. Psychiatric illnesses as oscillatory connectomopathies. Neuropsychopharmacology. 2016;41:387-8.

\title{
NMDA-receptor independent actions of ketamine: a new chapter in a story that's not so old
}

\author{
Nathan H. Wray ${ }^{1,2}$ and Mark M. Rasenick ${ }^{1,2}$ \\ Neuropsychopharmacology (2019) 44:220-221; https://doi.org/10.1038/s41386-018-0201-y
}

It has been nearly two decades since ketamine was introduced as a rapid acting antidepressant with good clinical efficacy in subjects who failed to remit in response to more conventional therapies [1]. Given it's potential for abuse and a litany of deleterious consequences in long-term ketamine users, serious questions remain about the utility of ketamine therapy. Yet, for many with intractable depression, ketamine provides considerable benefits. Hence the question: is there some substance that has the actions of ketamine, without being ketamine. To answer this, we must determine how ketamine works as an antidepressant.

Ketamine carries the epithet, "NMDAR antagonist." However, high-throughput screens have shown that most drugs in common use have multiple targets, and ketamine is no exception. Furthermore, many NMDAR antagonists have entered clinical trials for depression, but none have displayed the rapid, robust and long-acting antidepressant effects of ketamine [2]. (R)Ketamine has a fourfold decrease in affinity for the NMDAR compared to its (S)-enantiomer yet shows stronger and longerlasting antidepressant effects in preclinical models of depression, strengthening the hypothesis that a NMDAR-independent mechanism may be responsible for much of ketamine's antidepressant action [1]. A metabolite derived from $(R)$-ketamine also displays antidepressant effects in murine models of depression independent of the NMDAR [1]. These findings are tempered by the apparent antidepressant efficacy of esketamine in clinical trials. To address these apparent contradictions and to parse the molecular sites of ketamine action, we have turned to a simple cellular system with a straightforward biological reporter for antidepressant action.

Using this model system, we recently identified one NMDARindependent mechanism that may contribute to ketamine's antidepressant effects. Every antidepressant examined thus far translocates $\mathrm{Ga}_{\mathrm{s}}$ from lipid rafts to the non-raft membrane regions, where it enjoys a more facile and productive relationship with adenylyl cyclase, increasing cAMP production. $\mathrm{Ga}_{\mathrm{s}}$ translocation can be assayed directly, by cellular fractionation, or indirectly by determining mobility of a fluorescent $\mathrm{Ga}_{\mathrm{s}}$ with fluorescence recovery after photobleaching (FRAP) and/or by measuring augmented CAMP production [3-5]. While most antidepressants require a 3-day incubation with cells to achieve this effect, a 15min treatment of ketamine was sufficient to translocate $\mathrm{Ga}_{\mathrm{s}}$ from lipid rafts to non-raft regions. This "antidepressant biosignature" also included increased FRAP and elevated CAMP. Associated downstream subcellular events consistent with elevated CAMP; phosphorylation of cAMP related proteins and expression of BDNF were also evoked by $15-\mathrm{min}$ ketamine treatment. Ketamine produced similar results between 1 and $10 \mu \mathrm{M}$. The former reflects plasma concentrations in patients and the latter, tissue concentrations in rodent studies. The increase in CAMP was maintained after near complete elimination of the NMDAR within the cells.

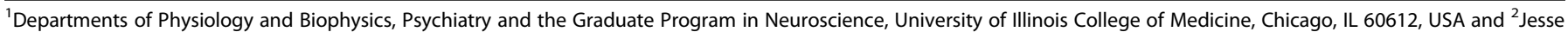
Brown VAMC, Chicago, IL 60612, USA

Correspondence: Mark M. Rasenick (raz@uic.edu)

Published online: 13 September 2018 